
\title{
R Research Square \\ Forecasting the COVID-19 Outbreak: An Application of Arima and Fuzzy Time Series Models
}

\section{Prashant Verma}

State Bank of India

Mukti Khetan ( $\nabla$ mukti.khetan11@gmail.com )

Indian Institute of Technology Bombay

Shikha Dwivedi

University of the Chinese Academy of Sciences

Shweta Dixit

GD Goenka University

\section{Research}

Keywords: Coronavirus, COVID-19, ARIMA, FUZZY Time Series, Forecasting

Posted Date: June 24th, 2020

DOI: https://doi.org/10.21203/rs.3.rs-36585/v1

License: (9) This work is licensed under a Creative Commons Attribution 4.0 International License. Read Full License 


\title{
FORECASTING THE COVID-19 OUTBREAK: AN APPLICATION OF ARIMA AND FUZZY TIME SERIES MODELS
}

\author{
Prashant Verma ${ }^{1}$ Mukti Khetan ${ }^{2}$ Shikha Dwivedi $^{3}$ and Shweta Dixit ${ }^{4}$
}

\author{
${ }^{1}$ Analytics Department Global IT Centre, SBI, Navi Mumbai, India \\ Email: prashantvermag@gmail.com \\ ${ }^{2 *}$ Department of Mathematics, Indian Institute of Technology Bombay, Mumbai Maharashtra, India \\ Email: mukti.khetan11@gmail.com \\ ${ }^{3}$ Department of Physics, University of Chinese Academy of Sciences, Beijing, China \\ Email: shikhadwij@gmail.com \\ ${ }^{4}$ School of Management, GD Goenka University, Gurugram, Haryana, India \\ Email: sdshwetadixit@gmail.com
}

\begin{abstract}
Purpose: The whole world is surfaced with an inordinate challenge of mankind due to COVID19, caused by 2019 novel coronavirus (SARS-CoV-2). After taking hundreds of thousands of lives, millions of people are still in the substantial grasp of this virus. This virus is highly contagious with reproduction number $\mathrm{R}_{0}$, as high as 6.5 worldwide and between 1.5 to 2.6 in India. So, the number of total infections and the number of deaths will get a day-to-day hike until the curve flattens. Under the current circumstances, it becomes inevitable to develop a model, which can anticipate future morbidities, recoveries, and deaths.

Methods: We have developed some models based on ARIMA and FUZZY time series methodology for the forecasting of COVID-19 infections, mortalities and recoveries in India and Maharashtra explicitly, which is the most affected state in India, following the COVID-19 statistics till "Lockdown 3.0" (17 $7^{\text {th }}$ May 2020).

Results: Both models suggest that there will be an exponential uplift in COVID-19 cases in the near future. We have forecasted the COVID-19 data set for next seven days. The forecasted values are in good agreement with real ones for all six COVID-19 scenarios for Maharashtra and India as a whole as well.

Conclusion: The forecasts for the ARIMA and FUZZY time series models will be useful for the policymakers of the health care systems so that the system and the medical personnel can be prepared to combat the pandemic.
\end{abstract}

Keywords: Coronavirus; COVID-19; ARIMA; FUZZY Time Series; Forecasting.

\section{Background}

No one could ever presume the prevailing scenario that a minuscule RNA structure can ingest millions of lives and still starved to have many more millions, leaving no stone unturned. The world, utterly equipped with leading-edge technology, is experiencing a disaster in saving lives as well as in screening the economy. Thus, despite warnings of some researchers, no country 
was ever prepared for this confrontation with the imperceptible adversary of humanity. The ongoing pandemic is caused by severe acute respiratory syndrome coronavirus 2 (SARS-CoV2), also acknowledged as 2019 novel coronavirus (2019-nCov) or COVID-19 but publicly known as just "coronavirus", the name derived from the virus structure, where spike protein looks like a shape of a crown [1]. The full spectrum of COVID-19 ranges from the common cold, fever, mild respiratory complications to severe progressive pneumonia, multi-organ failure, and death. This virus is devilishly transmissible with the reproduction number $\left(\mathrm{R}_{0}\right)$ ranging from 1.5 to 6.5 globally, i.e., each infected individual can infect at least 1.5 other individuals [2,3]. Thus, each infection causes furthermore and the outbreak will continue to grow. The estimated number of $\mathrm{R}_{0}$ in India was 2.56 prior to the "Lockdown 2.0 " $\left(30^{\text {th }}\right.$ April 2020) [4] and it appears that Maharashtra has an elevated $R_{0}$, as there, growth in the number of COVID-19 victims is intense, particularly, in two of its robust cities, Mumbai and Pune.

Even now, it is un-conceivable that this virus has spread its deadly wings around the whole world and gripped all 218 countries into its lethal clutches in such a short span of time. Thus far, over five million human beings have been infected, and more than 0.3 million lives have been lost worldwide [5]. In this era, many economic, medical, political, and cultural activities, as well as the tourism enterprises of various countries, are interconnected with each other, which led the virus to spread so fast. India also made no exemption and got its first COVID-19 case as early as on the $30^{\text {th }}$ of January 2020 in Kerala. At the initiation stage, the dissemination was slow as only three COVID-19 cases were identified for more than a month but after that, morbidities got an exponential surge and then took no halt since then. Until the $3^{\text {rd }}$ national lockdown i.e. "Lockdown 3.0" (17 $7^{\text {th }}$ May 2020), almost 0.1 million confirmed cases had been reported in India, of which over 3000 succumbed to the infection [6]. With these statistics, India is confronting a grave threat of COVID-19 disease and set foot into the list of the top 10 most infected countries of the world. Maharashtra is the worst affected state of India, which alone accounts for one-third of the country's total cases.

The ultimate solution to this crisis is the successful development of the vaccine. However, as of now, the only alternative is to contain the disease, preventing its further diffusion in society. In this connection, we need some temporary makeshifts to properly handle the morbidities with currently available options as the startling increase in COVID-19 cases is putting tremendous pressure on the medical services, leading to a shortage of intensive care resources throughout the country. Under such background, the utmost requirement is to establish a realistic and computationally effective methodology that can successfully forecast the potential morbidities, and thus, it can assist in decision making and logistical planning in healthcare systems for the following imminent challenges. These models may curtail the anticipated chaos for health care workers. The statistical prediction models are always conducive to prediction and in this way, it may restrain the comprehensive plight in the pandemic.

In the present work, we have used the Auto-Regressive Integrated Moving Average (ARIMA) model [7] and the fuzzy time series (FTS) model [8] for determining the forthcoming number of COVID-19 cases of India as a whole, along with India's worst-hit state, Maharashtra. The rationale behind the choice of the ARIMA model is the following: 
i. This model gives remarkably satisfying results in the prediction of natural adversities, when compared to other prediction models, such as wavelet neural network (WNN) model and support vector machine (SVM) model [9].

ii. ARIMA model has been used earlier in a similar crisis like situation, as during the SARS outbreak, Earnest et al. [10] exercised this model to make a real-time prediction on the number of beds occupied in Tan Tock Seng Hospital, Singapore. Also, Anwar et al. [11] used the ARIMA model to develop a predictive tool for malaria patterns in Afghanistan. Recently, Benvenuto et al. [12] employed this model to anticipate the forthcoming COVID-19 morbidities globally.

Although limited research has been done on FTS methodology in health and epidemiology fields, yet it can be an alternative way to forecast the COVID-19 sepsis, as for some study, the FTS model proved itself more precise than ARIMA model. For instance, Wang [13] shows that the FTS model can be utilized to predict export values (from Taiwan) accurately, outperforming the ARIMA model. Similar conclusions have been drowned by Zhang et al. [14] in the analysis of infectious disease surveillance and by Tricahya and Rustam [15] in forecasting the number of pneumonia patients in Jakarta.

We have implemented these two methodologies and COVID-19 stats from the Indian Government database [6] (up to "Lockdown 3.0") for calculations and forecasted the COVID19 infections, mortalities, and recoveries. The paper is organized as follows: In Section 2, we recount the details of ARIMA and FTS models used in this work. It is followed by the results of modeling and a detailed analysis of the outcome, in Section 3. Finally, conclusions are drawn in Section 4.

\section{Material and methods}

This study utilizes the COVID 19 data obtained from the 'Ministry of Health and Family Welfare, Government of India' concerning new cases/infections, mortalities, and recoveries[6]. In order to forecast the COVID 19 scenarios in India, classical time series (ARIMA) and fuzzy time series have been utilized. The core difference between the classical time series and the fuzzy time series is that the values of the former are real numbers while the values of the latter are fuzzy sets.

\subsection{ARIMA model}

ARIMA models (Box-Jenkins, [7]) are the extensively used technique for time series forecasting, characterizing the autocorrelations in the data. The algorithm of this model is as follows:

\subsubsection{Identification of stationary time series:}


a) The first step in developing the Box-Jenkins model is to determine whether the data is stationary or not. If nonstationarity exists, it can be modeled by differencing it to an appropriate level of difference. The Augmented Dickey-Fuller (ADF) test is performed to test the stationarity of the data. If the p-value is greater than 0.05 , we reject the null hypothesis $\left(\mathrm{H}_{0}\right.$ : unit root is present, i.e., non-stationary) otherwise it will be accepted at a 5\% level of significance.

b) Identification of parameters: The next step is to identify the parameters $p$ and $q$ of the $\operatorname{ARMA}(p, q)$ model by using two diagnostic plots, autocorrelation function (ACF) and partial autocorrelation function (PACF). For a given, non-stationary time series $X_{t}$, the ARIMA (p, d, q) model can be written as:

$$
\phi(B)(1-B)^{d} X_{t}=\psi(B) \varepsilon_{t}
$$

Where,

d: Number of times, need to differentiate $X_{t}$, to make the data stationary.

B: Backward shift operator.

Hence,

$\phi(B): 1-\phi_{1} B-\phi_{2} B^{2}-\ldots-\phi_{p} B^{p}$

$\psi(B): 1+\psi_{1} B+\psi_{2} B^{2}+\ldots+\psi_{p} B^{p}$ are characteristic polynomials in B and $\varepsilon_{t}$ is the white noise, where, $\varepsilon_{t} \sim \mathrm{N}(0,1)$

2.1.2 Parameter estimation: $R$ 3.6.1 software has been used to find coefficients that are best fitted for the selected ARIMA model. The selection of the ARIMA model is done by the minimum Akaike information criterion (AIC), [16]. It is written as

$$
\mathrm{AIC}=-2 \log (\mathrm{L})+2(\mathrm{p}+\mathrm{q}+\mathrm{k})
$$

Where $\mathrm{L}$ is the likelihood of data, $\mathrm{p}$ is the order of autoregressive part, $\mathrm{q}$ is the order of moving average part, and $\mathrm{k}$ represents the intercept of the ARIMA model.

2.1.3 Diagnostic check: This process involves testing of the assumptions of models: whether the parameters achieve statistical significance or multicollinearity and whether the residual term is white noise or not. Ljung- Box tests are performed to test the adequacy, and ACF of the residuals are plotted. The aforementioned steps are reiterated until the required adequacy is achieved.

2.2 Fuzzy time series model: The concept of the fuzzy time series was first developed by Song and Chisson [8]. The main advantage of the FTS technique is that there are no assumptions considered for the data set. Here, we briefly reviewed some concepts of FTS from $[8,17]$.

\section{Definition 1 (Fuzzy Set) [18]}

Let $\mathrm{U}$ be the universe of discourse, where $U=\left\{u_{1}, u_{2}, \ldots u_{n}\right\}$. A fuzzy set $A_{i}$ of $\mathrm{U}$ is defined by $A_{i}=f_{A_{i}}\left(u_{1}\right) / u_{1}+f_{A_{i}}\left(u_{2}\right) / u_{2}+\ldots+f_{A_{i}}\left(u_{n}\right) / u_{n}$, where $f_{A_{i}}$ is the membership function of fuzzy 
set $A_{i}, f_{A_{i}}: U \rightarrow[0,1] . u_{k}$ is the element of fuzzy set $A_{i}$, and $f_{A_{i}}\left(u_{k}\right)$ is the degree of belongingness of $u_{k}$ to $A_{i} \cdot f_{A_{i}}\left(u_{k}\right) \in[0,1]$ where $1 \leq k \leq n$. The definition of FTS is briefly reviewed as follows:

\section{Definition 2 (FTS) [8, 17]}

Let $Y(t)(t=\ldots, 0,1,2 \ldots)$, is a subset of R. Let $Y(t)$ be the universe of discourse defined by fuzzy set $f_{i}(t)$. If $F(t)$ collection of $f_{i}(t)(i=1,2, \ldots), F(t)$ is defined as a fuzzy time series on $Y(t)(\mathrm{t}=\ldots, 0,1,2, \ldots)$.

\section{Definition 3 (Fuzzy logical relationship) $[8,17,19]$}

If there exists a fuzzy relationship $R(t-1, t)$, such that $F(t)=F(t-1) \times R(t-1, t)$ where $\mathrm{x}$ represents an operator, then $F(t)$ is said to be caused by $F(t-1)$. When $F(t-1)=A_{i}$ and $F(t)=A_{j}$, the relationship between $F(t-1)$ and $F(t)$ is called a fuzzy logical relationship (FLR) and denoted by $A_{i} \rightarrow A_{j}$.

\section{Definition 4 (fuzzy logical relationship group) $[8,17,19]$}

Fuzzy logical relationships with the same fuzzy set on the left-hand side can be further grouped into fuzzy logical relationship groups. Suppose there are fuzzy logical relationships such that

$$
\begin{aligned}
& A_{i} \rightarrow A_{j 1}, \\
& A_{i} \rightarrow A_{j 2}, \\
& A_{i} \rightarrow A_{j m},
\end{aligned}
$$

They can be grouped into a fuzzy logical relationship group (FLRG) $A_{i} \rightarrow A_{j 1}, A_{j 2}, \ldots A_{j m}$ We have estimated the forecast values of COVID-19 data sets by following Chen's model [19], but the length of the interval is defined as the algorithm average based length given by Hurang [20].

The algorithm of FTS model is presented as follows:

Step 1 Define the universe: The universe of discourse $U\left[L_{c}-D_{1}, U_{c}+D_{2}\right]$, where $L_{c}$ and $U_{c}$ are the lower and Upper limit of the data respectively and $\mathrm{D}_{1}$, and $\mathrm{D}_{2}$ are the constants which we get using $10 \%$ of the $\mathrm{L}_{\mathrm{c}}$ and $\mathrm{U}_{\mathrm{c}}$. So, we have extrapolated the upper and lower bounds by $10 \%$ as a security margin.

Step 2 Divide the universe of discourse into intervals: The Universe of discourse is partitioned into ' $l$ ' length of the interval, where ' $l$ ' is based on algorithm 'average based length' by Hurang (2001). Using this length ' $l$ ', we divide the universe of discourse into intervals.

Step 3 Fuzzify the time series data set: Achieve $u_{1}, u_{2}, \ldots u_{n}$, and define fuzzy sets $A_{i}$; $A_{1}, A_{2}, \ldots A_{n}$ and fuzzify the data. 
Step 4 Identify the Fuzzy Logical Relationship and Fuzzy Logical Relationship Group: Identification of the FLRs and FLRGs based on Definition 3 and 4 respectively.

Step 5 Defuzzify and compute the forecasted values: We have used the algorithm of Chen's model [19] for forecasting and result are presented in Table 2.

Step 6 Validation: Examine the performance analysis parameter using Root Mean Square Error [RMSE]. The RMSE can be defined as

$$
R M S E=\sqrt{\frac{\sum_{i=1}^{n}\left(\text { Forcast }_{i}-\text { Actual }_{i}\right)^{2}}{n}}
$$

\section{Results and Discussions}

The data sets utilized in the forecasting, are collected from various Indian databases [6], which contain total COVID-19 morbidities, recoveries, and deaths, reported in India and also contain data subsets for the state of Maharashtra throughout the phase between $2^{\text {nd }}$ March 2020 to $17^{\text {th }}$ May 2020. In Figure 1, we have plotted six distinct datasets for daily cases of COVID-19 infections, recoveries, and deaths recorded in India and Maharashtra, which shows an exponential growth for all the six data sets. Thus we can say that all the series are nonstationary. We have also conducted the Augmented Dickey-Fuller / Unit root test to prove that these six data sets are not stationary.

Figure 1: Graphical Representation of COVID-19 statistics in India and Maharashtra

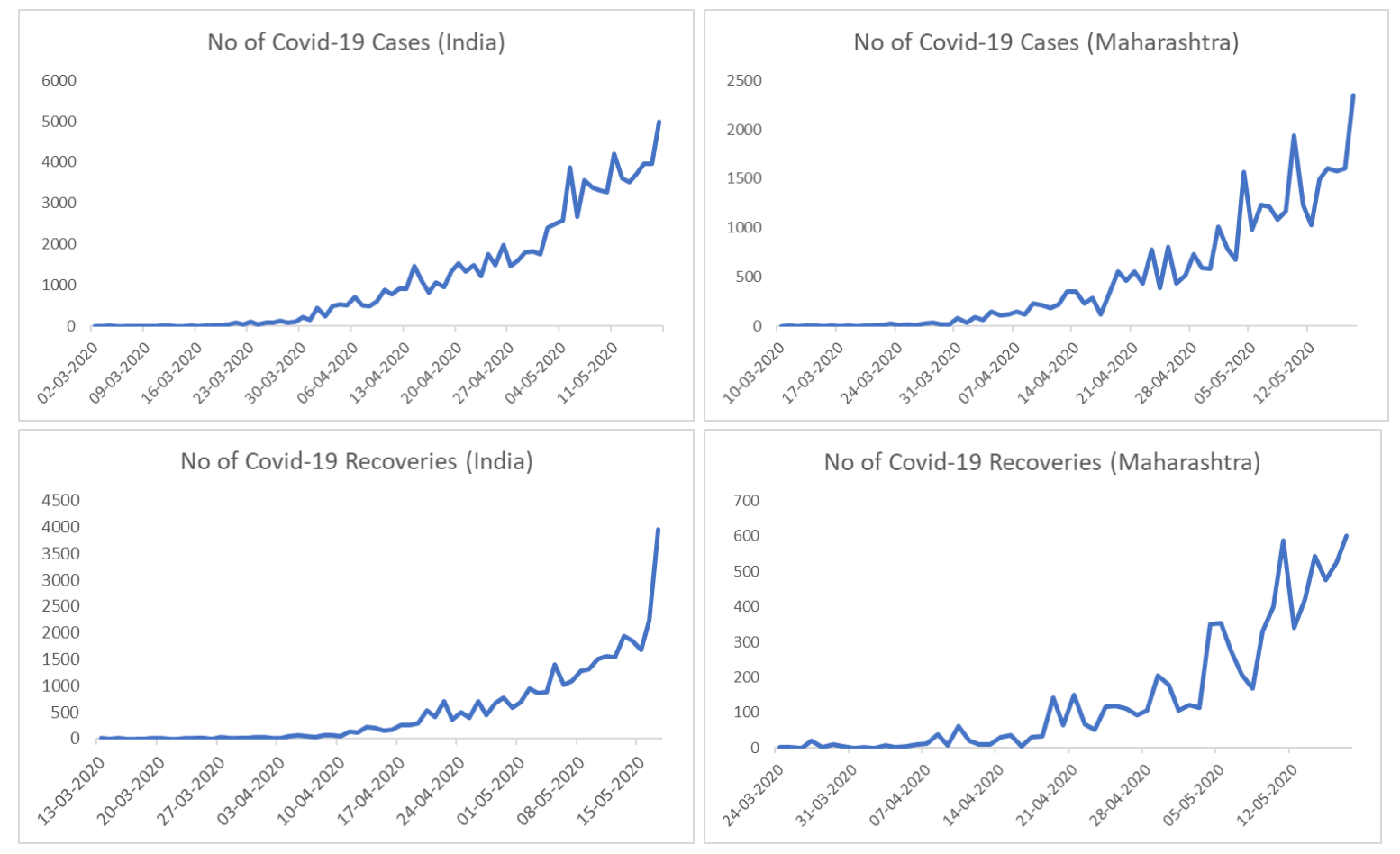



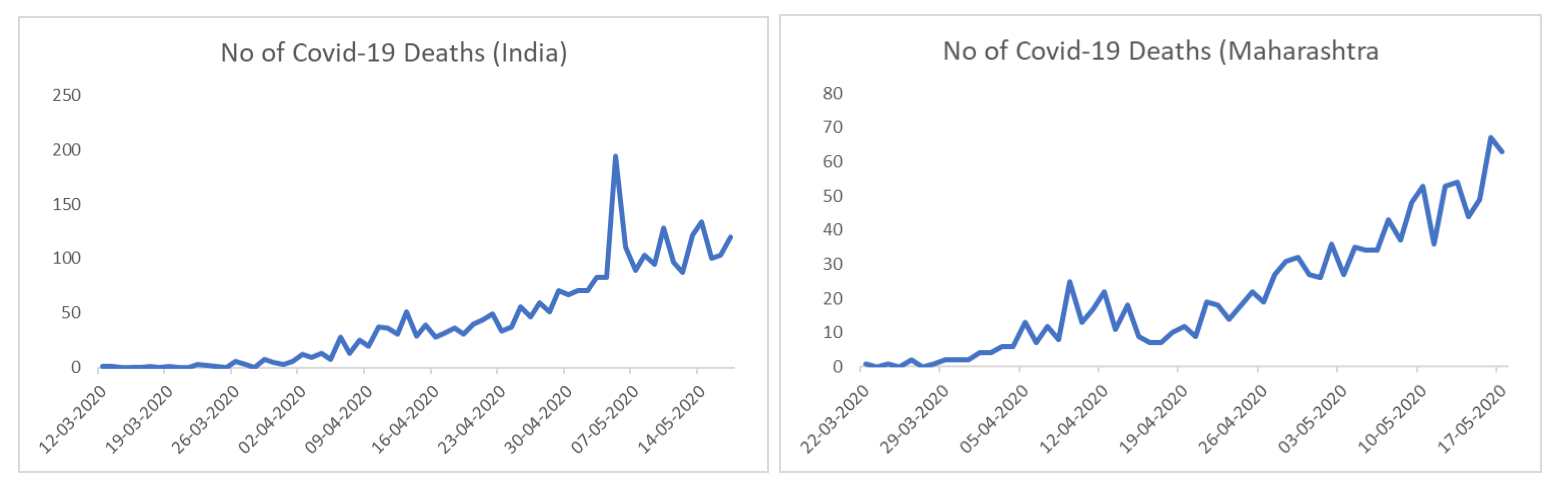

\subsection{Unit root test/ADF Test}

We have performed an ADF test to check the stationarity of data and results of the test are summarized in Table 1 showing p-values corresponding to different datasets. According to the ADF test, $\mathrm{p}$-value $>0.05$, leads to a non-stationary model, and as it can be seen from the table, that the $\mathrm{p}$-value corresponding to each data sample, is greater than 0.05 , implying that all the series are non-stationary, which validates the conclusion from previous graphs.

Table 1: Unit Root Test of the six data sets of COVID-19

\begin{tabular}{lcc}
\hline Data set & Test statistic value & P-value \\
\hline India & & \\
\hline No of cases & -0.31422 & 0.9876 \\
\hline No of recovery & 3.09040 & 0.9900 \\
\hline No of deaths & -2.06880 & 0.5472 \\
\hline Maharashtra & & \\
\hline No of cases & 0.024982 & 0.9900 \\
\hline No of recovery & -0.69697 & 0.9652 \\
\hline No of deaths & -0.59160 & 0.9744 \\
\hline
\end{tabular}

3.2 Autocorrelation function and Partial Autocorrelation function: ACF and PACF of the data have been used for assessing the combination of ARIMA models. We have used this method to find the values of parameters $p$ and $q$.

3.3. ARIMA and FTS model fitting: The best fitted ARIMA and FTS models for COVID-19 datasets of India and Maharashtra along with the estimated parameters are summarized in Table 2. The diagnostic measures AIC and RMSE are also mentioned. From this table, it is clear that among the daily cases of COVID-19 infections and recoveries in India particularly in Maharashtra, the ARIMA model appears to be a more suitable model as it gives lower RMSE value compared to FTS model. Similarily, ARIMA predicts better for the case of COVID-19 
deaths in India. However, in the case of COVID-19 deaths in Maharashtra, the FTS model is the best-fitted model for forecasting because of its lower RMSE value.

Table 2: Best fitted model of COVID-19 data sets with parameter estimation, diagnostic measure, and RMSE values

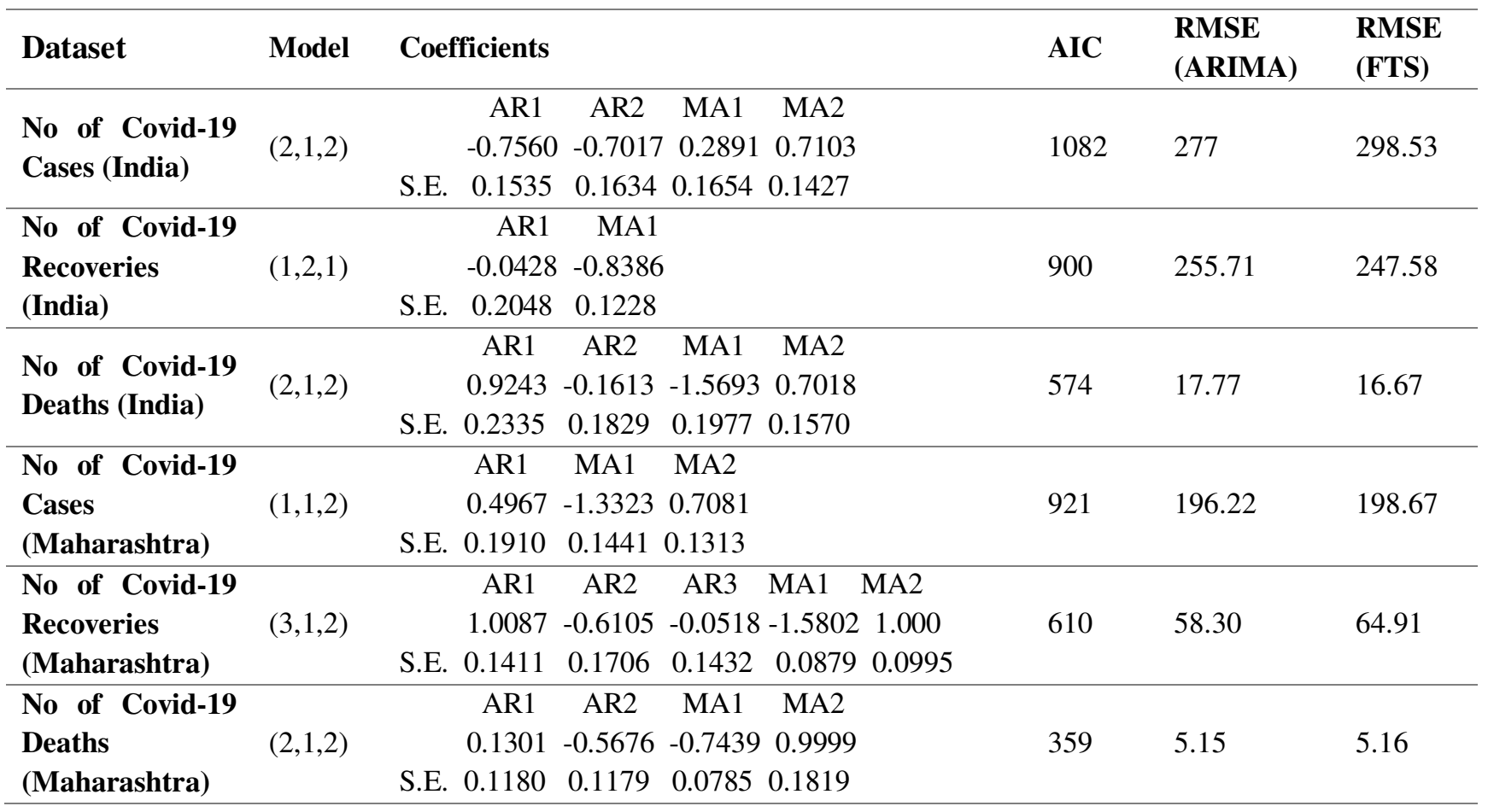

\subsection{Diagnostic Check}

3.4.1 Ljung Box test for residuals: The Box-Ljung test [7] is a diagnostic tool used to test the lack of fit of a time series model. In the following Table 3, we have shown the values of the Ljung-Box test statistic along with its corresponding $\mathrm{p}$-value for all the six data sets.

Table 3: Ljung-Box Test for COVID-19 data

\begin{tabular}{lcc}
\hline \multicolumn{1}{c}{ Dataset } & $\begin{array}{c}\text { Ljung Box test } \\
\text { statistic }\end{array}$ & p-value \\
\hline No of Covid-19 Cases (India) & 17.888 & 0.5299 \\
\hline No of Covid-19 Recoveries (India) & 16.590 & 0.6177 \\
\hline No of Covid-19 Deaths (India) & 9.0866 & 0.9720 \\
\hline No of Covid-19 Cases (Maharashtra) & 22.232 & 0.2729 \\
\hline $\begin{array}{l}\text { No of Covid-19 Recoveries } \\
\text { (Maharashtra) }\end{array}$ & -0.69697 & 0.9652 \\
\hline No of Covid-19 Deaths (Maharashtra) & 11.736 & 0.8966 \\
\hline
\end{tabular}

Table 3 provides relevant validation $(\mathrm{p}>0.5)$ in favor of the Null Hypothesis at $5 \%$ level of significance among all the six cases establishing the suitability of the models. 
3.4.2 Residual ACF: As we know, the primary measure of goodness of fit is that the errors of the fitted models should be uncorrelated. This is the required condition which ensures that the model fitting is appropriate. We have plotted the residual ACFs in Figure 2, where black spikes in between the horizontal dotted blue lines (control band) are the residual ACFs. If the spikes of residual ACF are on both sides of the horizontal axis in a random pattern and gradually decreases to zero, we may call it an ideal situation. Eventually, as the lag increases, spikes fall inside the control band. Hence, it is clear from Figure 2 that all ARIMA models are fitted appropriately.

Figure 2: Graphical representation of residual ACF of COVID-19 data samples

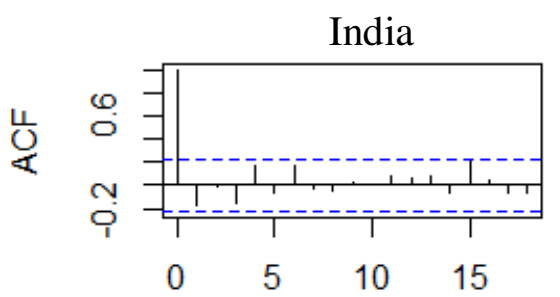

Lag

ACF graph of Covid-19 Cases in India

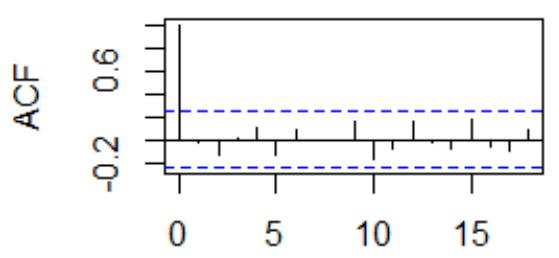

Lag

ACF graph of Covid-19 Recoveries in India

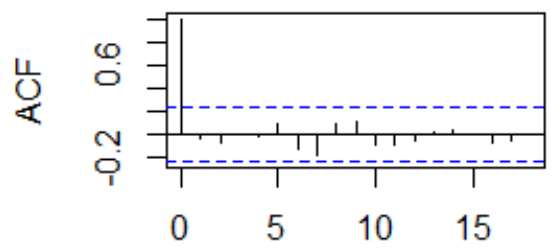

Lag

ACF graph of Covid-19 Deaths in India

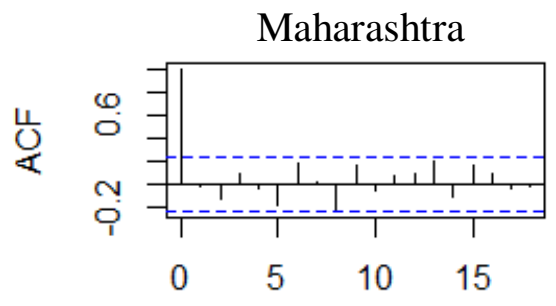

Lag

ACF graph of Covid-19 Cases in Maharashtra

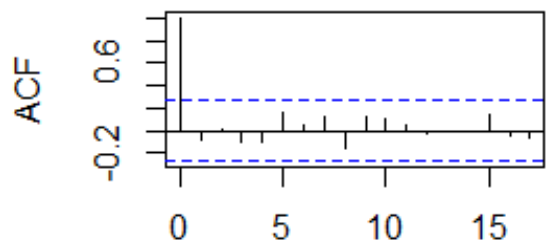

Lag

ACF graph of Covid-19 Recoveries in Maharashtra

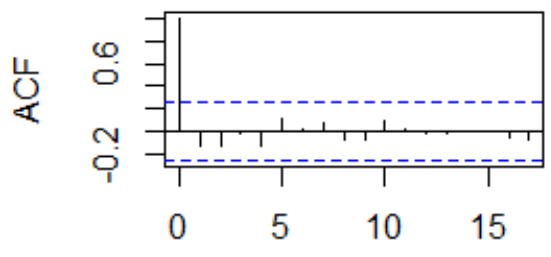

Lag

ACF graph of Covid-19 Deaths in Maharashtra

\subsection{Forecasting}

The forecasted values of COVID-19 infections, recoveries, and deaths in India and Maharashtra respectively, have been compiled in Table 4. The prediction is based on the best-fitted model with minimum RMSE. Thus, in cases of daily COVID-19 infections and recoveries in India and in Maharashtra along with the COVID-19 deaths in India, we have shown the prediction of ARIMA models in terms of lower (Lo) and upper (Hi) predictive intervals and FTS forecast are shown for the cases of COVID-19 deaths in Maharashtra. 
Table 4: Forecasted values of COVID-19 pandemic based on ARIMA and FTS model

\begin{tabular}{cccccc}
\hline \multicolumn{7}{c}{ No. of Cases (India) } \\
\hline Date & Forecast & Lo 80 & Hi 80 & Lo 95 & Hi 95 \\
\hline $\mathbf{1 8 - 0 5 - 2 0 2 0}$ & 4574.929 & 4217.354 & 4932.504 & 4028.065 & 5121.793 \\
\hline $\mathbf{1 9 - 0 5 - 2 0 2 0}$ & 4616.351 & 4211.141 & 5021.561 & 3996.635 & 5236.066 \\
\hline $\mathbf{2 0 - 0 5 - 2 0 2 0}$ & 4874.169 & 4357.882 & 5390.457 & 4084.576 & 5663.763 \\
\hline $\mathbf{2 1 - 0 5 - 2 0 2 0}$ & 4650.194 & 4032.384 & 5268.004 & 3705.335 & 5595.053 \\
\hline $\mathbf{2 2 - 0 5 - 2 0 2 0}$ & 4638.619 & 3978.005 & 5299.234 & 3628.296 & 5648.942 \\
\hline $\mathbf{2 3 - 0 5 - 2 0 2 0}$ & 4804.524 & 4078.981 & 5530.067 & 3694.902 & 5914.146 \\
\hline $\mathbf{2 4 - 0 5 - 2 0 2 0}$ & 4687.221 & 3892.622 & 5481.819 & 3471.987 & 5902.455 \\
\hline
\end{tabular}

\begin{tabular}{cccccc}
\hline \multicolumn{7}{c}{ No. of Recoveries (India) } \\
\hline Date & Forecast & Lo 80 & Hi 80 & Lo 95 & Hi 95 \\
\hline $\mathbf{1 8 - 0 5 - 2 0 2 0}$ & 4269.975 & 3937.176 & 4602.773 & 3761.004 & 4778.946 \\
\hline $\mathbf{1 9 - 0 5 - 2 0 2 0}$ & 4644.256 & 4144.924 & 5143.588 & 3880.594 & 5407.918 \\
\hline $\mathbf{2 0 - 0 5 - 2 0 2 0}$ & 5015.956 & 4360.713 & 5671.198 & 4013.848 & 6018.063 \\
\hline $\mathbf{2 1 - 0 5 - 2 0 2 0}$ & 5387.766 & 4578.021 & 6197.511 & 4149.368 & 6626.164 \\
\hline $\mathbf{2 2 - 0 5 - 2 0 2 0}$ & 5759.572 & 4793.296 & 6725.847 & 4281.780 & 7237.363 \\
\hline $\mathbf{2 3 - 0 5 - 2 0 2 0}$ & 6131.377 & 5005.033 & 7257.722 & 4408.782 & 7853.973 \\
\hline $\mathbf{2 4 - 0 5 - 2 0 2 0}$ & 6503.183 & 5212.493 & 7793.874 & 4529.243 & 8477.124 \\
\hline
\end{tabular}

\begin{tabular}{cccccc}
\hline & \multicolumn{5}{c}{ No. of Cases (Maharashtra) } \\
\hline Date & Forecast & Lo 80 & Hi 80 & Lo 95 & Hi 95 \\
\hline $\mathbf{1 8 - 0 5 - 2 0 2 0}$ & 1783.557 & 1530.237 & 2036.877 & 1396.137 & 2170.977 \\
\hline $\mathbf{1 9 - 0 5 - 2 0 2 0}$ & 2047.780 & 1791.059 & 2304.501 & 1655.159 & 2440.401 \\
\hline $\mathbf{2 0 - 0 5 - 2 0 2 0}$ & 2179.017 & 1897.348 & 2460.685 & 1748.242 & 2609.792 \\
\hline $\mathbf{2 1 - 0 5 - 2 0 2 0}$ & 2244.201 & 1923.770 & 2564.631 & 1754.144 & 2734.257 \\
\hline $\mathbf{2 2 - 0 5 - 2 0 2 0}$ & 2276.577 & 1913.332 & 2639.821 & 1721.042 & 2832.111 \\
\hline $\mathbf{2 3 - 0 5 - 2 0 2 0}$ & 2292.657 & 1887.179 & 2698.136 & 1672.531 & 2912.783 \\
\hline $\mathbf{2 4 - 0 5 - 2 0 2 0}$ & 2300.644 & 1855.080 & 2746.209 & 1619.212 & 2982.077 \\
\hline
\end{tabular}

\begin{tabular}{cccccc}
\hline & \multicolumn{5}{c}{ No. of Recoveries (Maharashtra) } \\
\hline Date & Forecast & Lo 80 & Hi 80 & Lo 95 & Hi 95 \\
\hline $\mathbf{1 8 - 0 5 - 2 0 2 0}$ & 589.8314 & 513.1324 & 666.5304 & 472.5304 & 707.1325 \\
\hline $\mathbf{1 9 - 0 5 - 2 0 2 0}$ & 577.4383 & 493.2116 & 661.6650 & 448.6246 & 706.2520 \\
\hline $\mathbf{2 0 - 0 5 - 2 0 2 0}$ & 567.2070 & 480.6949 & 653.7190 & 434.8983 & 699.5156 \\
\hline $\mathbf{2 1 - 0 5 - 2 0 2 0}$ & 564.9792 & 474.5108 & 655.4477 & 426.6197 & 703.3388 \\
\hline $\mathbf{2 2 - 0 5 - 2 0 2 0}$ & 569.6203 & 468.8292 & 670.4115 & 415.4735 & 723.7671 \\
\hline $\mathbf{2 3 - 0 5 - 2 0 2 0}$ & 576.1919 & 459.2873 & 693.0964 & 397.4018 & 754.9819 \\
\hline $\mathbf{2 4 - 0 5 - 2 0 2 0}$ & 580.1027 & 447.3557 & 712.8498 & 377.0836 & 783.1219 \\
\hline
\end{tabular}




\begin{tabular}{|c|c|c|c|c|c|c|}
\hline \multirow[b]{2}{*}{ Date } & \multicolumn{5}{|c|}{ No. of Deaths (Maharashtra) } & \multirow{2}{*}{\begin{tabular}{|c|}
$\begin{array}{c}\text { No. of Deaths } \\
\text { (India) }\end{array}$ \\
Forecast (FTS)
\end{tabular}} \\
\hline & $\begin{array}{l}\text { Forecast } \\
\text { (ARIMA) }\end{array}$ & Lo 80 & Hi 80 & Lo 95 & Hi 95 & \\
\hline $18-05-2020$ & 59.76160 & 52.98000 & 66.54319 & 49.39004 & 70.13315 & 134.137 \\
\hline 19-05-2020 & 64.80111 & 57.50464 & 72.09757 & 53.64213 & 75.96009 & 97.5540 \\
\hline $20-05-2020$ & 67.29481 & 58.51826 & 76.07136 & 53.87223 & 80.71738 & 105.683 \\
\hline 21-05-2020 & 64.75885 & 53.22811 & 76.28959 & 47.12410 & 82.39359 & 109.748 \\
\hline 22-05-2020 & 63.01353 & 49.73721 & 76.28986 & 42.70914 & 83.31792 & 91.4570 \\
\hline 23-05-2020 & 64.22586 & 50.04374 & 78.40798 & 42.53618 & 85.91554 & 103.651 \\
\hline 24-05-2020 & 65.37420 & 50.23762 & 80.51079 & 42.22479 & 88.52362 & 109.748 \\
\hline
\end{tabular}

$\mathrm{L}_{0}, \mathrm{H}_{\mathrm{i}}$ : lower and upper predictive intervals with the risk error (size of the critical region): $\alpha=0.2,0.05$

Figure 3: Graphical representation of Forecasted values of the COVID-19 pandemic with ARIMA and FTS Model

Forecasts from ARIMA(2,1,2)

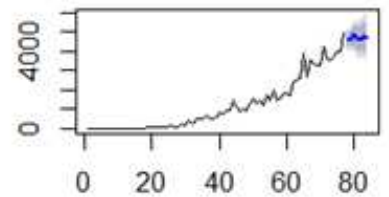

Forecast of Covid-19 Cases in India

Forecasts from ARIMA $(1,2,1)$

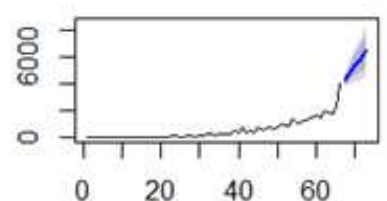

Forecast of Covid-19 Recoveries in India

\section{Forecasts from FTS}

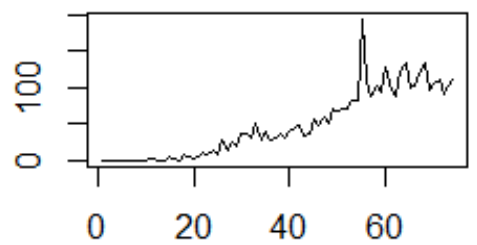

Forecast of Covid-19 Deaths in India
Forecasts from ARIMA(1,1,2)

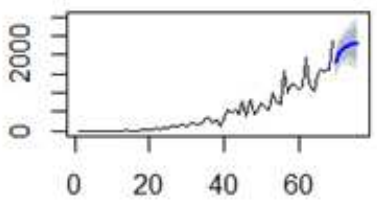

Forecast of Covid-19 Cases in Maharashtra

\section{Forecasts from ARIMA $(3,1,2)$}

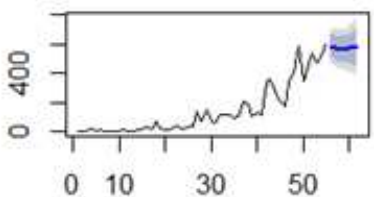

Forecast of Covid-19 Recoveries in Maharashtra

\section{Forecasts from ARIMA(2,1,2)}

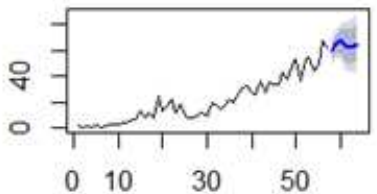

Forecast of Covid-19 Deaths in Maharashtra 
In Figure 3 we have plotted the forecasts of COVID-19 outbreak in India and Maharashtra resulted from ARIMA models (5 sets) and FTS model (1 set), using data as of Table 4. In this figure, the dark blue line shows the actual forecasted values whereas the ensemble of light blue lines gives the range of estimated values between the lower and upper predictive intervals.

Figure 4: Comparison between the actual and forecasted values of COVID-19 data sets

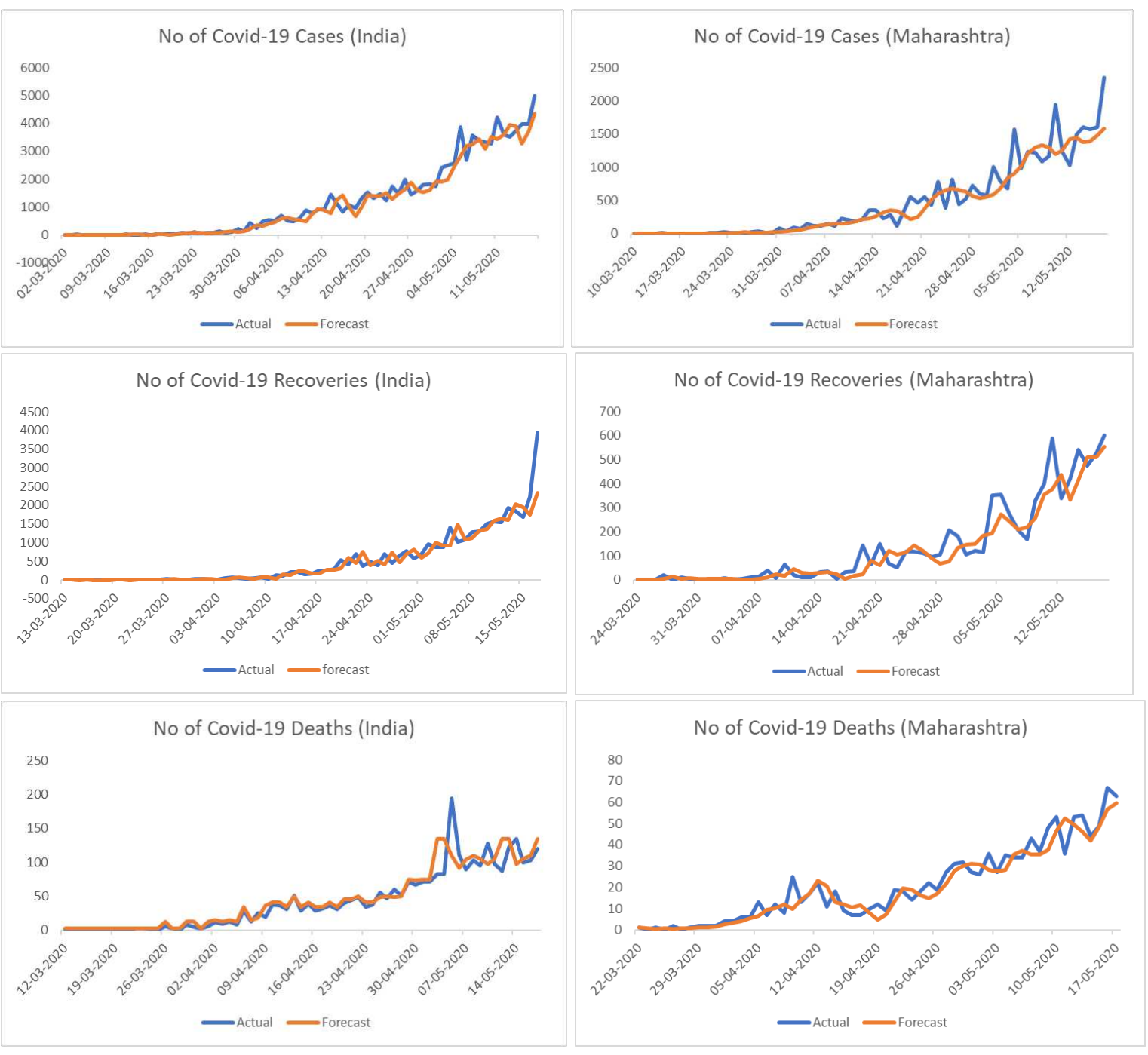

In Figure 4, we have plotted the actual values against the forecasted values of the parameter concerning COVID-19 pandemic data with ARIMA and FTS model. We have utilized RStudio (Version 1.2.5) for forcasting COVID-19 data through ARIMA model and Python (Version 3.7) through the Fuzzy Time Series model.

\section{Conclusions}

We have used univariate time series models (ARIMA and FTS) to estimate all aspects of the looming COVID-19 infections, recoveries, and deaths in India and its most vulnerable state of the ongoing pandemic, Maharashtra, based on contemporary statistics of gratis Indian data 
banks. In this rationale, first, we have modeled three different COVID-19 non-stationary samples of infections, recoveries, and deaths in India and Maharashtra separately. Thereafter, we have fitted the model parameters in accordance with both ARIMA and FTS methodology. The calculated RMSE values of these models are utilized as conclusive tools to demonstrate befitting models for all six COVID-19 data sets concerning three parameters, incidence, mortality, and recovery. We have reported the estimated range of COVID-19 figures by ARIMA models, including randomized risk error factors (Figure 3). This report shows that hereafter the risk of contamination in India is not likely to decrease, particularly in Maharashtra, we may see an intensified scenario. Although the speculated overall recovery rate in India holds an optimistic trend for the upcoming days as the recovery rate is considerably higher than the number of new infections.

Nevertheless, this is not the case for Maharashtra, where the number of new infections will increase sharply and the recovery rate will not be so satisfactory in the following time. Death predictions are concluded by the ARIMA model explicitly in Maharashtra and by the FTS model in India as a whole (Figure 3). In both cases, the average mortality rate is persistent, under the global average.

We have compared the forecasted values with the actual statistics of COVID-19 till "Lockdown 3.0" to assess the potential of our models. Figure 4 depicts the forecasted values are in good agreement with real ones for all six COVID-19 scenarios for Maharashtra and India as a whole as well. However, the figure exhibits a few instances where we have observed some fluctuations between the observed and forecasted number of COVID-19 infections and recoveries on day to day basis. Our models may face this limitation in a few cases because of the following constraints:

1. ARIMA and FTS models reviewed the pandemic by considering the time variations of only one variable, while a number of effective tools that possess a role in the picture of correlation of COVID-19 data sets were extended in subsequent lockdowns such as testing capacity enhanced at least tenfold and still continue to increase.

2. Despite lockdown, millions of people have been migrated from their workplaces to hometowns, of which many found infected on arrival. So, these people were either the earlier undetected cases or they got infected during their journey.

3. Many infected individuals, particularly asymptomatic or mild ones are still not reported to the healthcare systems.

As per the findings of the current study, the risk of a boom in the infection rate will be higher in the coming days. Also, as of now, "Lockdown 4.0" is much more relaxed than the previous lockdowns, hereafter we will notice an uninterrupted growth in total COVID-19 cases of India and Maharashtra. This is an alarming state of affairs for the policymakers to practice better resources in prevention and control of the further transmission. Our models may assist them to estimate new infections and to plan the required strategy for supplying potential health facilities to all individuals and for balancing the burden of public health care systems as the majority of hospitals of India are established in urban areas only. This preparatory preparation will heal 
the current adversity, protect human lives, and prevent heavy economic losses. Realizing the crucial importance of such modeling, we are planning to extend this work and use different multivariate models for forecasting, which will provide a further precise description of the current pandemic situation.

List of abbreviations: Not applicable.

\section{Declarations}

Ethics approval and consent to participate: Not applicable.

Consent for publication: Not applicable.

Availability of data and material: Data are available on www.mohfw.gov.in, www.covid19india.org

Competing interests: "The authors declare that they have no competing interests".

Funding: Not applicable.

Authors' contributions: Prashant Verma participated in the coding using R and python. Mukti khetan participated in the design of the study and performed the statistical analysis. Shikha Dwivedi reviewed the literature and drafted the manuscript. Shweta Dixit participated in the theoretical section of ARIMA and FUZZY TIME SERIES model. All authors read and approved the final manuscript.

Acknowledgements: Not applicable.

References

1. Tyrrell D.A. and Fielder M. (2002). Cold Wars: The Fight Against the Common Cold. Oxford UniversityPress. p. 96. ISBN 978-0-19-263285-2

2. James Holland Jones (2007). Califonia: Department of Anthropological Sciences.

3. Kevin Linka, Mathias Peirlinck, Ellen Kuhl, Reproduction number of COVID-19 and how it relates to public health measure, Department of Mechanical Engineering, Stanford University, Stanford, California, United States, medRxiv preprint DOI: https://doi.org/10.1101/2020.05.01.20088047.

4. Balram Rai, Anandi Shukla, Laxmi Kant Dwivedi, COVID-19 in India: Predictions, Reproduction Number and Public Health Preparedness, Department of Mathematical Demography and Statistics, International Institute for Population Sciences, Mumbai 400088 medRxiv preprint DOI: https://doi.org/10.1101/2020.04.09.20059261.

5. Website: www.worldometers.info/coronavirus/

6. Websites: www.mohfw.gov.in, www.covid19india.org

7. Box, G. E. P., and G. M. Jenkins (1976). Time Series Analysis: Forecasting and control. Rev. ed. San Francisco: Holden-Day 
8. Song Q. and Chissom B.S. (1993). Forecasting enrollments with fuzzy time series-part I, Fuzzy Sets Syst 54(1):1-9.

9. Zhang Y., Yang H., Cui H., and Chen Q. (2019). Comparison of the ability of ARIMA, WNN and SVM models for drought forecasting in the sanjiang Plain, China, Nat. Resour. Res., 29, 1447.

10. . Earnest, A., Chen, M. I., Ng, D., and Sin, L. Y. (2005). Using autoregressive integrated moving average (ARIMA) models to predict and monitor the number of beds occupied during a SARS outbreak in a tertiary hospital in Singapore. BMC Health Services Research, 5(1), 36.

11. Anwar, M. Y., Lewnard, J. A., Parikh, S., and Pitzer, V. E. (2016). Time series analysis of malaria in Afghanistan: using ARIMA models to predict future trends in incidence, Malaria Journal, 15(1), 566.

12. Benvenuto D., Giovanetti M., Vassallo L., Angeletti S. and Ciccozzi M. (2020). Application of the ARIMA model on the COVID- 2019 epidemic dataset, Data in brief 29:105340.

13. Wang C.C. (2011). A comparison study between fuzzy time series model and ARIMA model for forecasting Taiwan export, Expert Systems with Applications 38:9296-9304.

14. Zhang T., Zhang X., Liu Y., Luo Y., Zhou T., and Li X. (2016). The analysis of infectious disease surveillance data based on fuzzy time series method, International Journal of Infectious Diseases, 45, 309-310.

15. Tricahya S. and Rustam Z. (2019, June). Forecasting the Amount of Pneumonia Patients in Jakarta with Weighted High Order Fuzzy Time Series. In IOP Conference Series: Materials Science and Engineering (Vol. 546, No. 5, p. 052080). IOP Publishing.

16.Akaike H. (1974) A New Look at the Statistical Model Identification, IEEE Transactions on Automatic Control, AC- 19, 716-723.

17. Song Q., Chissom BS (1994) Forecasting enrollments with fuzzy time series-part II, FuzzySets Syst 62(1):1-8.

18. Zadeh L.A. (1965). Fuzzy sets, Inf Control 8(3):338-353.

19. Chen S.M. (1996). Forecasting enrollments based on fuzzy time series, Fuzzy Sets Syst 81:311319.

20. Huarng K. (2001). Effective lengths of intervals to improve forecasting in fuzzy time series, Fuzzy Sets Syst 123:387-394. 
Figures
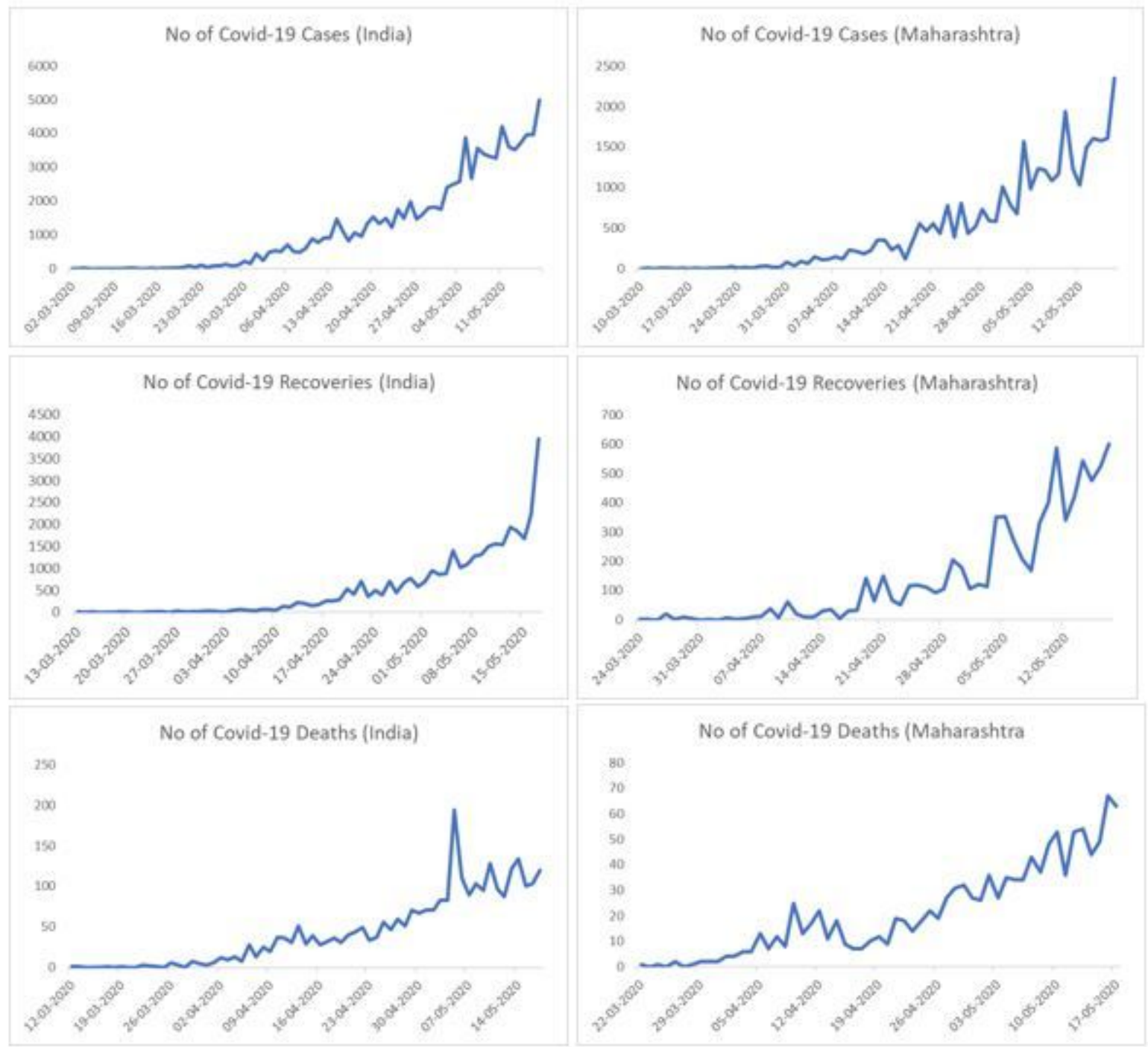

\section{Figure 1}

Graphical Representation of COVID-19 statistics in India and Maharashtra 


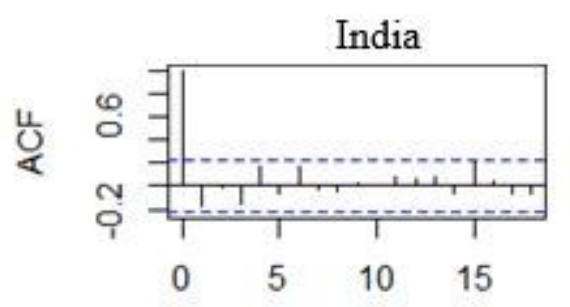

Lag

ACF graph of Covid-19 Cases in India

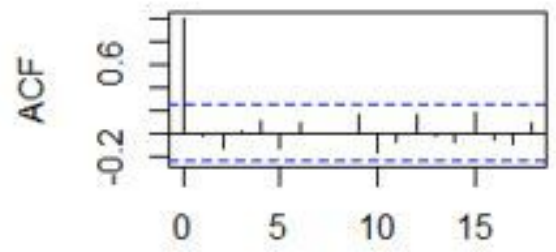

Lag

ACF graph of Covid-19 Recoveries in India

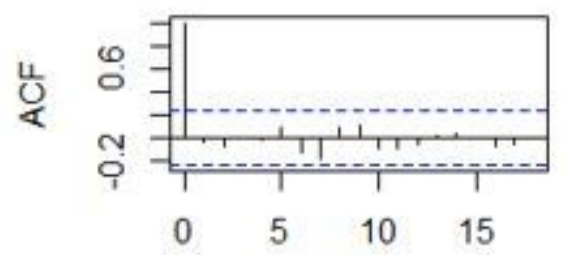

Lag

ACF graph of Covid-19 Deaths in India

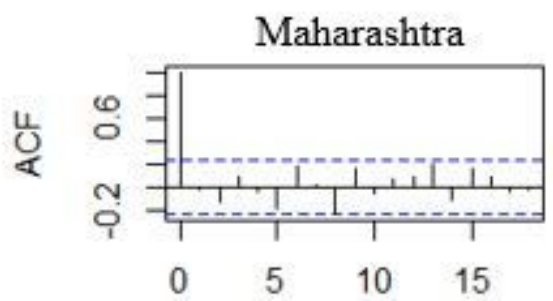

Lag

ACF graph of Covid-19 Cases in Maharashtra

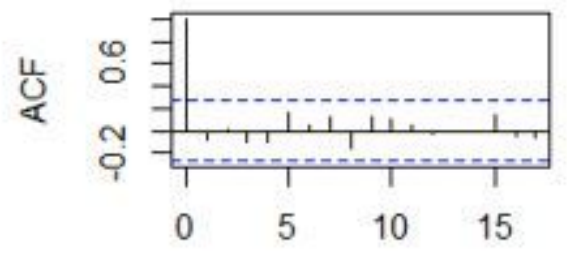

Lag

ACF graph of Covid-19 Recoveries in Maharashtra

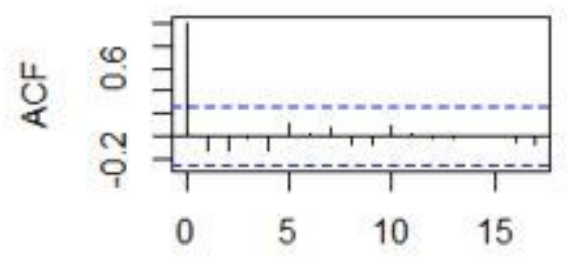

Lag

ACF graph of Covid-19 Deaths in Maharashtra

Figure 2

Graphical representation of residual ACF of COVID-19 data samples 
Forecasts from ARIMA(2,1,2)

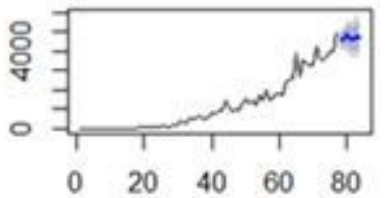

Forecast of Covid-19 Cases in India

Forecasts from ARIMA(1,2,1)

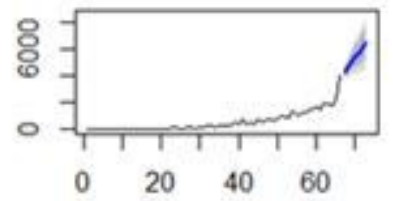

Forecast of Covid-19 Recoveries in India

\section{Forecasts from FTS}

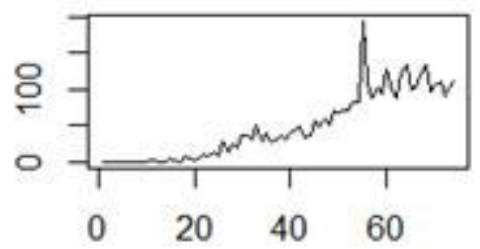

Forecast of Covid-19 Deaths in India
Forecasts from ARIMA(1,1,2)

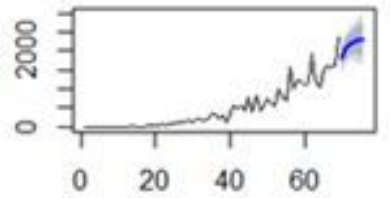

Forecast of Covid-19 Cases in Maharashtra

\section{Forecasts from ARIMA $(3,1,2)$}

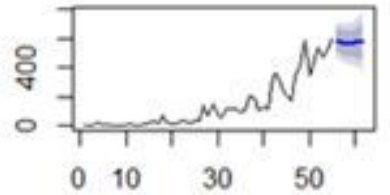

Forecast of Covid-19 Recoveries in Maharashtra

Forecasts from ARIMA(2,1,2)

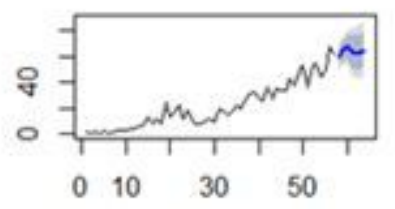

Forecast of Covid-19 Deaths in Maharashtra

\section{Figure 3}

Graphical representation of Forecasted values of the COVID-19 pandemic with ARIMA and FTS Model 

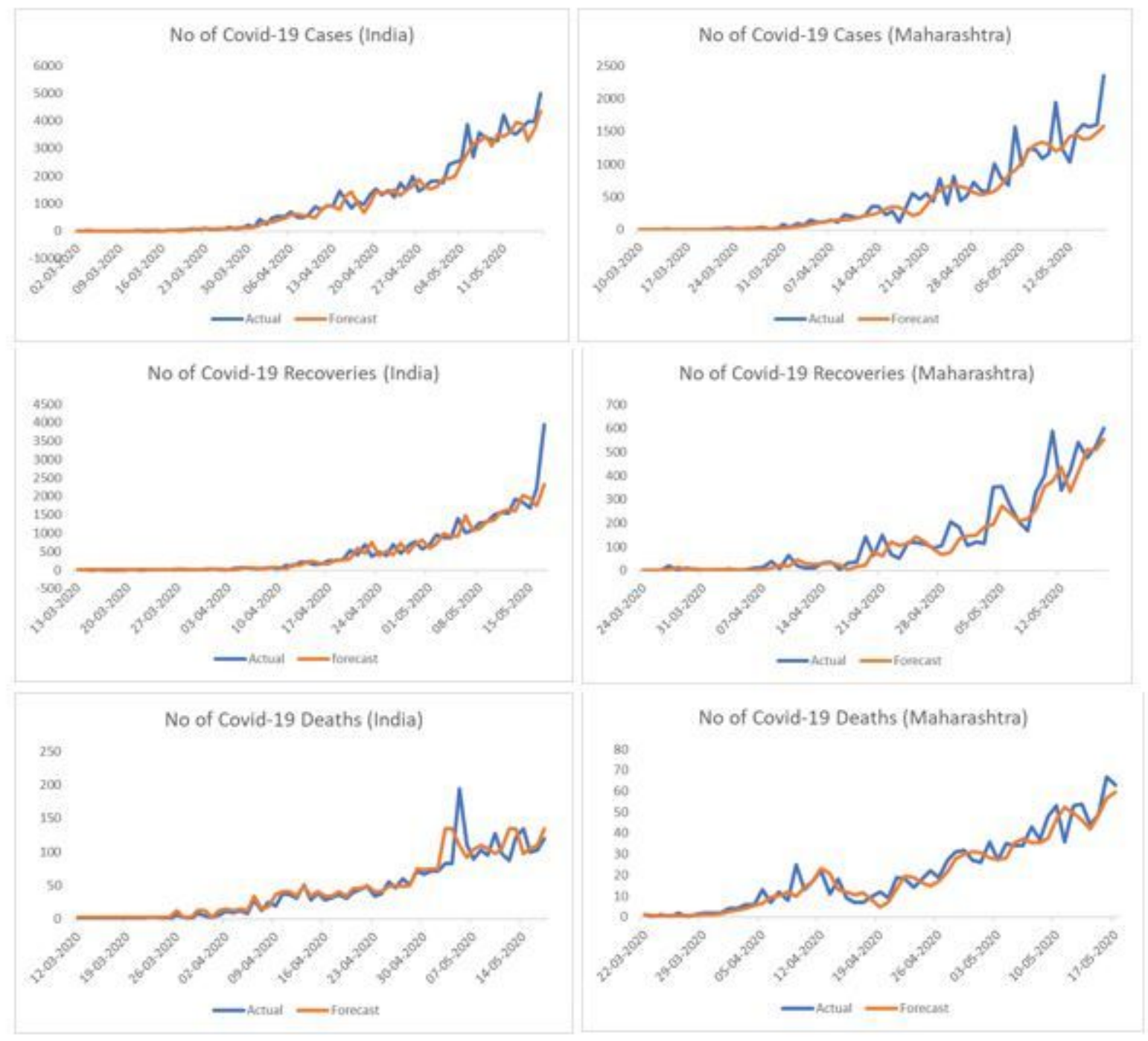

\section{Figure 4}

Comparison between the actual and forecasted values of COVID-19 data sets 